\title{
Research on seamless development of surgical instruments based on biological mechanisms using CAD and 3D printer
}

\author{
Ikuo Yamamoto ${ }^{\text {a, }}$, Ren Ota ${ }^{\mathrm{b}}$, Rui Zhu ${ }^{\mathrm{a}}$, Murray Lawn ${ }^{\mathrm{d}}$, Takakazu Ishimatsu ${ }^{\mathrm{a}}$, Takeshi \\ Nagayasu $^{\mathrm{c}}$, Naoya Yamasaki ${ }^{\mathrm{c}}$, Katsunori Takagi ${ }^{\mathrm{c}}$ and Takehiko Koji ${ }^{\mathrm{d}}$ \\ ${ }^{a}$ Department of Mechanical Science, Nagasaki University Graduate School, 1-14 Bunkyomachi, \\ Nagasaki-shi, Nagasaki 852-8521, Japan \\ ${ }^{b}$ Department of Engineering, Nagasaki University, 1-14 Bunkyomachi, Nagasaki-shi, Nagasaki \\ 852-8501, Japan \\ ${ }^{c}$ Department of Surgery, Nagasaki University Graduate School of Biomedical Sciences, 1-7-1 \\ Sakamotomachi, Nagasaki-shi, Nagasaki 852-8501, Japan \\ ${ }^{d}$ Nagasaki University Graduate School of Biomedical Sciences, 1-7-1 Sakamotomachi, Nagasaki-shi, \\ Nagasaki 852-8501, Japan
}

\begin{abstract}
In the area of manufacturing surgical instruments, the ability to rapidly design, prototype and test surgical instruments is critical. This paper provides a simple case study of the rapid development of two bio-mechanism based surgical instruments which are ergonomic, aesthetic and were successfully designed, prototyped and conceptually tested in a very short period of time.
\end{abstract}

Keywords: 3D-printer, CAD simulation, rapid prototyping, surgical instruments

\section{Introduction}

In an age of rapid advances in medical technology the need to be able to rapidly prototype such as medical instruments has become of increasing importance [1,2]. Recently, there has been an increasing need for surgical instruments that can hold organs delicately yet stably. Such an instrument increases the efficacy of surgical operations by decreasing the physical and mental strain on both surgeons and patients. New bio-mechanism based surgical instruments based in part on the anatomical structure of a fish, provide soft handling forceps [3-5] where pressure is distributed over a larger area. The authors have used a seamless design and prototyping process. This process has been used to prototype biologically based mechanisms using 3D-CAD and a 3D-printer. Specifically, a fish based

\footnotetext{
* Address for correspondence: Ikuo Yamamoto, Department of Mechanical Science, Nagasaki University Graduate School, 1-14 Bunkyomachi, Nagasaki-shi, Nagasaki 852-8521, Japan. Tel.: +81-95-819-2512; Fax: +81-95-819-2534; E-mail: iyamamoto@nagasaki-u.ac.jp.
} 
mechanism which produces an elastic oscillating fin and shark skin which effectively reduces hydrodynamic resistance has been found to be effective in creating superior surgical instruments [6-8]. Classical surgical instruments which exert a large amount of force on a limited number of points on organs are stressful for both surgeons and patients. Furthermore classical instrument's ergonomics often do not consider the surgeon and are often awkward and thus stressful to use.

The purpose of the research is to develop user-friendly surgical instruments that enable more efficient surgery. This process is effectively facilitated by using a more seamless design through to the prototyping process using 3D-CAD and a 3D-printer. The surgical instrument's design and concept prototyping are discussed below.

\section{Surgical instrument design}

The basic steps of the system design and prototyping are shown in Figure 1. First, the specifications, such as a surgical instrument's function, form, size, elasticity, material characteristics, are designed, to suit the needs of the specific instrument. Specifications of some parts may mean creating a new kind of engineering. For the kinetics of surgical instruments, motion simulation is carried out in CAD. Next, verification of resultant measurement data of the surgical instrument is carried out. In considering the design of medical instruments, aesthetics, ergonomics and functional efficacy must be considered. Techniques for data measurement of the design include strength modeling based on the target production materials. This process uses stereolithography (STL) data (3D) which may be directly sent to a 3D-printer thus making the rapid prototyping of surgical instruments possible. Based on the basic plans, assembly drawings are created. Complex mechanisms can be reduced to individual parts. Each individual part making up the mechanism is prototyped, finally a surgical instrument is created. Prototyped instruments undergo practical tests, and, if the functionality is satisfactory the process ends.

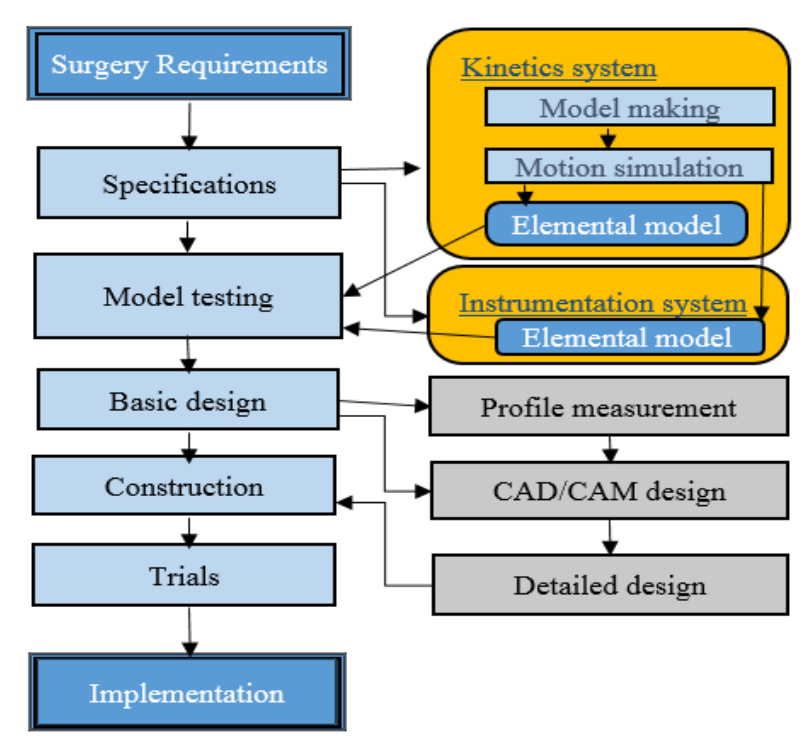

Fig. 1. Basic steps of design and prototyping. 


\section{Surgical instrument prototyping}

The developed surgical instruments based on these bio-mechanisms are shown in Figures 2 and 3. The length of the extractors is $295 \mathrm{~mm}$. Regarding the extractors, the instrument's shark skin based soft grasping mechanism makes them easy to use, allowing, a surgeon to operate smoothly despite the instrument becoming covered with fluids such as blood. The extractors' grip is stable on account of the shark skin based surface. This set of extractors shown in Figure 2 was prototyped in approximately 30 minutes. The STL data is sent to the 3D-printer, an Objet 260 Connex with maximum resolution of 16 microns. The soft grasping forceps designed are shown in Figures 4 and 5. It is estimated that the time required to manufacture this surgical instrument would be about 40 hours or so (CNC milling). The feedback to design is conducted rapidly. This allows for iterations as required prior to production of the final concept prototype.

Table 1 shows the specifications of the forceps, both target specifications and those achieved to date are shown. Figure 5 shows an approximated stress analysis (yield) using 3D-CAD (SolidWorks simulation) based on the strength of VeroWhite PolyJet resin. It must be noted the vertical struts in the target forceps' jaws should provide a degree of freedom at both ends to provide for an equal distribution of pressure along the jaw's surface, in order to excerpt even pressure on the organ being handled. This mechanism shown in Figure 3 reflects the design of the flexible oscillating fin used in to propel the robotic fish detailed in [6-8]. The prototyped product is shown in Figure 5 and production time is approximately 2.5 hours. The products are made by VeroWhite PolyJet resin material. It must be noted that for this particular application, the VeroWhite PolyJet resin lacked the tensile strength to provide fully functional medical instruments, however they enabled confirmation of the concept. In this regard a 3D printer capable of printing materials of higher tensile properties will need to be used such as powder based Laser Sintering (direct metal) or more traditional CNC milling technology $[1,2]$. In the case of the forceps the strength of the printed resin is sufficient for larger/ thicker parts, however for thinner parts or parts providing intricate mechanical functionality, parts with metallic strength will be required. For this experiment in the case of the forceps the size of intricate mechanical parts were scaled up to confirm the mechanical feasibility of the mechanism, thus the two sets of data in Figure 1, "Target" and "Experimental", other fundamental limitations include the maximum printable length (diagonal) and the ability of be able to physically remove the support material (photopolymer) produced by the $3 \mathrm{D}$ printer.

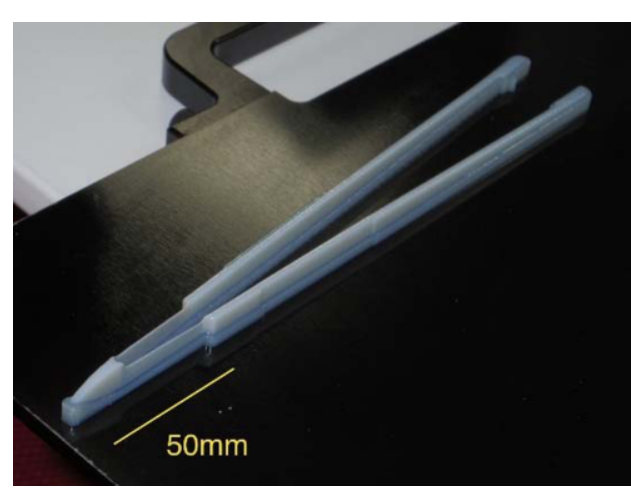

Fig. 2. 3D-printed extractor.

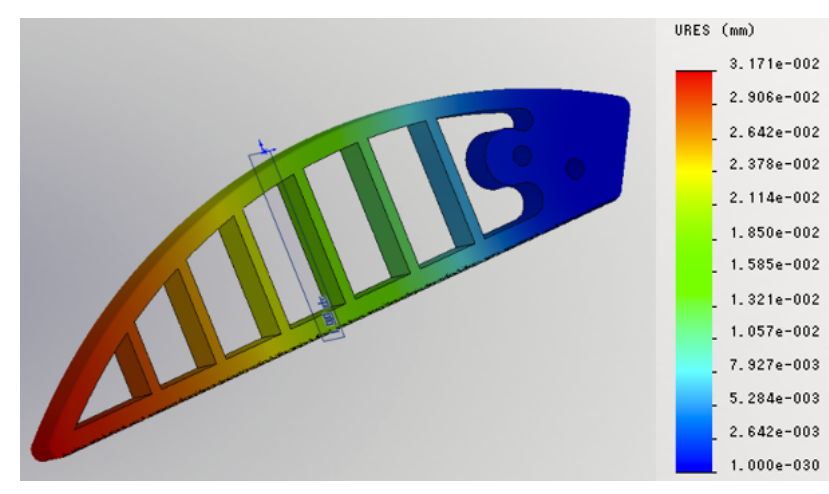

Fig. 3. Stress analysis of forceps' jaw. 
The authors have confirmed a seamless workflow from design through to conceptual prototype. An experimental test of the product is conducted shown in Figure 6.

\section{Conclusion}

A new basic method of seamless design through to prototyping has been utilized, which can significantly reduce time to production. In this study based on a bio-mechanism, an extractor and forceps which are ergonomic and aesthetic are successfully designed, prototyped and conceptually tested in a very short span of time.

Table 1

Specification of forceps

\begin{tabular}{|l|l|l|}
\hline Parameter & Target & Experimental \\
\hline Total length & $400 \mathrm{~mm}$ & $130 \mathrm{~mm}$ \\
\hline Hand grasp section length & $150 \mathrm{~mm}$ & $45 \mathrm{~mm}$ \\
\hline Tip length & $45 \mathrm{~mm}$ & $50 \mathrm{~mm}$ \\
\hline Tip thickness & $7 \mathrm{~mm}$ & $3 \mathrm{~mm}$ \\
\hline Overall tip thickness & $12 \mathrm{~mm}$ & $16 \mathrm{~mm}$ \\
\hline Opening angle (max) & 45 degrees & 45 degrees \\
\hline
\end{tabular}

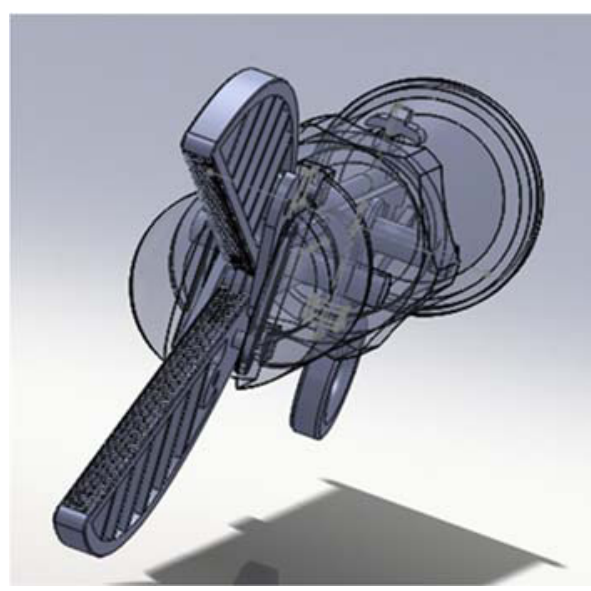

Fig. 4. CAD design of forceps.

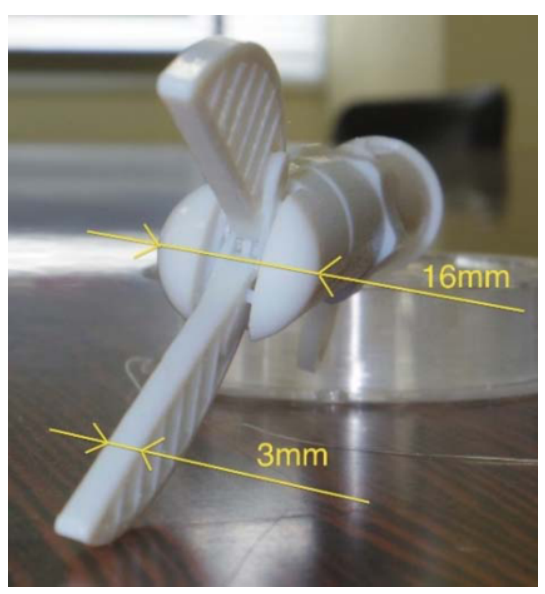

Fig. 5. 3D-printed forceps.

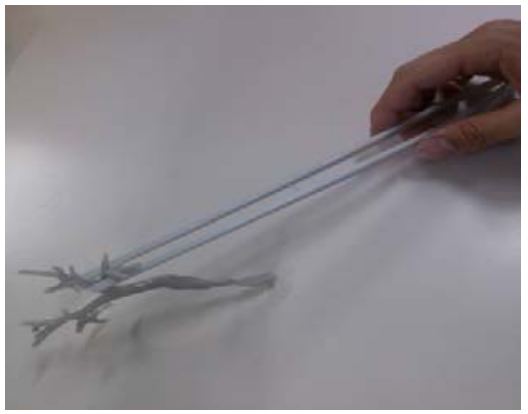

Fig. 6. Experimental test. 
In order to prototype fully functional instruments due to the limited strength of the 3D printer resin used more traditional manufacturing such as CNC milling or powder based Laser Sintering (direct metal) would be required particularly in the case of thin walled parts or parts providing intricate mechanical functionality.

The authors plan to make other surgical instruments, specifically, lung forceps based on a bio-flexible mechanism using this process. The 3D-printer is set up at the Hybrid Medic Training Center in the Nagasaki University Hospital, this enables surgeons to provide immediate feedback to engineering researchers by promptly evaluating the developed experimental production instruments.

\section{Acknowledgment}

A part of this study was supported by the Medical Engineering Hybrid professional development program staff. The authors would like to express their sincere gratitude to Mr. Naoto Matsuo, Mr. Nobuo Kakinoki, Nagasaki University and related personnel in this research and development.

\section{References}

[1] T.R. Kucklick, Rapid Prototyping for Medical Devices, In: The Medical Device R\&D Handbook, 2nd edi., Chapter 7 , CRC Press, New York, 2013, pp. 128-165.

[2] C.K. Chua, K.F. Leong and C.S. Lim, Rapid Prototyping-Principles and Applications, 3rd edi., World Scientific Publishing Co. Pte. Ltd., Singapore, 2010.

[3] Z. Gu, I. Yamamoto and N. Inagawa, Development of forceps robot for surgical operation by bio mechanism application, Proc. the 4th IEEE International Conference on Biomedical Engineering and Informatics (BMEI'11) Shanghai, China, 2011, pp. 1121-1126.

[4] Z. Gu, I. Yamamoto, N. Inagawa, T. Nakamura, K. Yamaguchi and K. Shibao, Modeling and analysis of flexible forceps robot for surgical operation, Proc. 2011 International Conference on Engineering and Business Management, China, 2011, pp. 3216-3218.

[5] Z. Gu, I. Yamamoto and N. Inagawa, Research and development of biomechanical robot for medical operation, Advanced Materials Research 452-453 (2012), 1121-1126.

[6] E. Madeen, Doctor Robopet, ANA Wingspan 416 (2004), 24-29.

[7] T. Ichikizaki and I. Yamamoto, Development of high performance robotic fish, Proceedings Techno-Ocean 2006 / 19th JASNAOE Ocean Engineering Symposium Kobe, Japan, October 18-20, 2006.

[8] I. Yamamoto, Development of robotic fish for the next generation unmanned marine vehicle, Further Advances in Unmanned Marine Vehicles, Chapter 16, IET Control, Engineering Series, 2012, pp. 359-372. 